\title{
The Results of Experimental Studies of Prestressed Pipelines under Operational Impacts
}

\author{
Alpisbay Imankulovich Ainabekov ${ }^{1}$, Ulanbator Seitkazievich Suleymenov ${ }^{1}$, Arman Berdibekovich \\ Moldagaliyev $^{1}$, Gaukhar Shapayevna Omashova ${ }^{1} \&$ Toremurat Tinisbekuly Serikbayev ${ }^{1}$ \\ ${ }^{1}$ South-Kazakhstan State University, Republic of Kazakhstan \\ Correspondence: Alpisbay Imankulovich Ainabekov, South-Kazakhstan State University, Republic of \\ Kazakhstan. E-mail: dinamika-nauka@rambler.ru
}

Received: December 26, 2014

Accepted: January 2, $2015 \quad$ Online Published: May 30, 2015

doi:10.5539/mas.v9n6p199

URL: http://dx.doi.org/10.5539/mas.v9n6p199

\begin{abstract}
Results of tests of prestressed pipeline models are presented in the article. Application of a way of preliminary stress for increase in static durability and improvement of dynamic characteristics of the main pipelines is experimentally proved. The formulas for determining the frequencies of free oscillations of the ring which is prestressed by a winding and the extended pipeline are offered. Received formulas consider of preliminary stress and service conditions. The test results showed that this technique can be used as a technique of active seismic protection of the design. This statement is based on the possibility of regulation by dynamic characteristics of designs by selection of a step and effort of a stress of a thread of a winding.
\end{abstract}

Keywords: dynamic characteristics, main pipeline, preliminary stress, parameters of a winding, dynamic influence

\section{Introduction}

Since design, construction and operation of pipelines are interfaced to large financial and material inputs, large metal consumption of pipelines, danger of environmental pollution, development of a network of pipelines have to be based on strictly scientific, technically feasible and economically justified decisions. The modern condition of designing and building of the main pipelines for oil and gas is characterized by continuous increase of pipes diameter, operating pressure at transportation and toughening of work of a construction.

In turn it imposes high degree of responsibility to the main pipelines, development of a construction of them should be based on essentially new, technically possible and economically defensible decisions. The design should prevent typical damage surveyed after major earthquakes.

Application of the prestressed technique, consisting of in a winding of high-strength wire, fiber glass or a tape on the case of pipes perpendicularly or angularly to form the pipeline can be considered as one of effective ways for perfections of a construction of the main pipelines.

The basic idea of such construction consists in:

- creation of pretensioned winding in the pipeline wall by force back sign with workers;

- redistribution effort for the purpose of decreasing of a wall thickness;

- creation of full-strength construction at the expense of smoothing of ring and longitudinal stress.

It is considered that created in a wall, as a result of a preliminary tension, tension go to a reserve of the bearing ability, increase durability and reliability, reduce metal consumption of the pipeline.

The way of preliminary stress by a winding of a high-strength profile on the case is widely applied in vessels and high-pressure apparatuses. Analysis of published data allowed to identify the following areas of research of prestressed pipelines:

- to study the behavior under static and dynamic loads;

- to study character of the intense deformed state at operational operating modes;

- to develop techniques of engineering calculation and optimum design. 
The existing norms of calculation on durability of the main pipelines don't regulate design calculations of previously strained pipeline at static and dynamic loadings.

The results of research of work features of prestressed pipelines at dynamic influence on the reduced models taking into account the operating conditions and various parametres of prestressed are discussed.

\section{Research Methods}

Experimental studies were conducted on models of the elevated pipelines made of low-carbonaceous steel with the accounting of opportunity and way of the appendix of loading, measurement of test parameters. According to recommendations about carrying out model tests of thin-walled metal designs modeling scale in 1:5 to natural designs of pipelines with a diameter of $1220 \mathrm{~mm}$ was increased. The general view of model is presented in Figure 1.

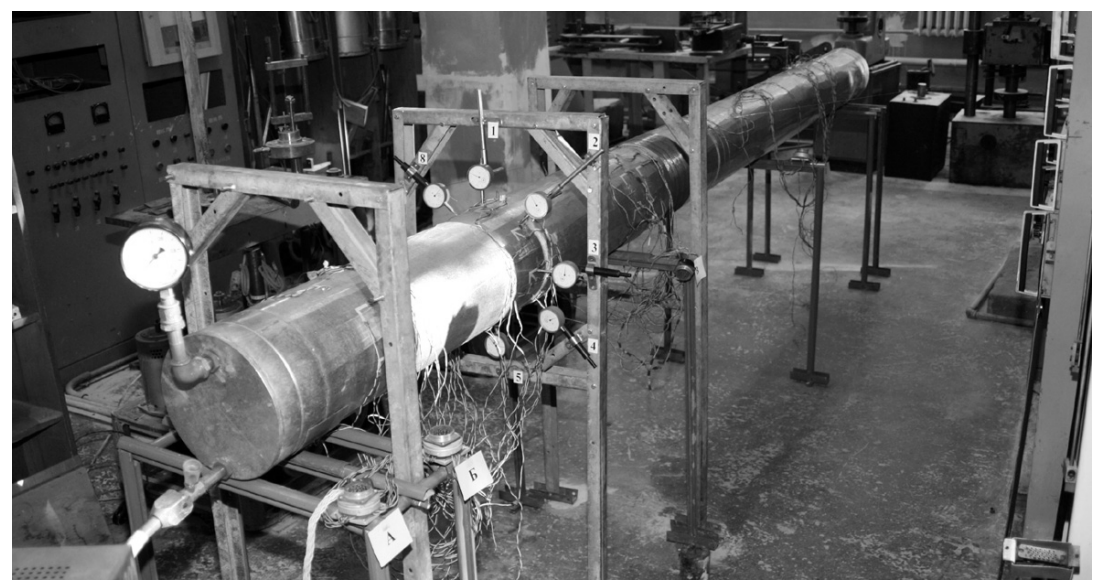

Figure 1. The general view of the pipeline model

Modeling of the geometry of the pipeline is made on the basis of simple mechanical similarity between model and a typical structural design. According to this theory, between model and nature parameters is established the constancy of scales of modeling. On a basis of the analysis of dimensions of physical quantities criteria and coefficients of similarity of model and the natural pipeline are established.

For the purpose of the full decision of experimental research were made three models of the fragment of the pipeline linear part: model A - base, without the prestressed, traditional designs, model B and C - prestressed, differing step of the winding wire, a winding (in model $\mathrm{B}$ to diameter of a wire $(a=d)$, in model $\mathrm{C}$ three diameters of a wire $(a=3 d))$.

The model represents a fragment of the pipeline linear part in length of $3980 \mathrm{~mm}$, consisting of three equal spans in length of $1260 \mathrm{~mm}$ and diameter $245 \mathrm{~mm}$ laid on support in height 600мм. The model by support condition corresponds to the pipeline with one restrained end in an end face, two free-movable support in the middle of span and one is longitudinal - a movable support in other end face. Cases of models are made of steel sheets of mark St8, and a winding wire - from steel St15. The windings on the model case was made by specially designed winding machine which allows to pull a wire with controllable stress and identical step on length of model.

As primary converters at measuring of relative deformations were applied single-element resistive-strain sensors. As the secondary measuring equipment served light sensor oscillograph complete with universal strain booster and a power unit. Measuring of dynamic movement was carried out by resistive-strain sensors with the ring transducer of movements.

Vibroelectrodynamic stand were used for implementation of dynamic tests of models of the pipeline in the work.

The following loadings were considered and modeled during implementation of test models: a) loading from weight of a transported product which was simulated by various filling of model with water (empty, partially filled and completely filled); b) loading from internal superfluous pressure which was created by charging in air model by the compressor and variants of test without superfluous pressure and with superfluous pressure equal $1,0 \mathrm{MPa}$ were considered; c) influence of prestressed which was simulated by winding of high-strength wire on the case of model with tensioning $0,75 S_{c r}$ and step $a=d$ and $a=3 d$ ( $S_{c r}$-critical force of instability of wall cover, $a$ - a step winding, $d$ - diameter of a wire). 
Dynamic tests were implemented in a mode of the free damped and forced resonance vibration, with the appendix of disturbing force in the middle of span model. For studying of spatial forms of fluctuations and amplitude-frequency characteristics of models is chosen the mode melting - staging of disturbing force. Frequency (period) and logarithmic decrement of oscillation were defined under the oscillogram of free oscillation of models.

\section{Results and Discussion}

\subsection{Results of Pilot Studies of Pipelines Models at Static Loadings.}

By experimental studies of models of pipelines on static loading it is found that as a result of the preliminary tension of the case of model of the pipeline ring stress in a wall of model decreased in 1,2 _. 3,6 times, and meridional stress increased in $2 \ldots 2,2$ times depending on service conditions and parameters of preliminary stress in comparison with tests of model of the pipeline traditional designs. At the same time deformations of a wall of previously strained pipeline, due to constraint of the case by winding decreased in $1,2 \ldots 4,2$ times depending on service conditions and parameters of preliminary stress. It is shown that the greatest effect from use of preliminary stress is reached at rather high levels of internal excessive pressure at which compatibility of work of a wall of the pipeline and the strained winding promotes some smoothing of the line of deformation and pipeline wall stress.

The form of a bend of a wall of models of the pipeline on flight in the lower point of section corresponds to a form of a bend of three flying rod systems of constant section.

\subsection{Results of Experimental Studies at Free-Oscillation Regime}

In experimental researches of models of the pipeline at its free-oscillation regime the task for the definition of frequencies and decrements of fluctuations taking into account various operating conditions and prestressed parameters, and also an estimation of influence on dynamic characteristics of prestressed model was assigned.

The analysis of oscillograms of damped free-oscillation model of prestressed pipeline has shown the qualitative influence of a wire winding on the absorption mechanism of energy oscillation that is proved by smoothness of decrease of a curve bending around amplitudes of the damped oscillation, looking like exponents.

It is established, that use of prestressed in the pipeline construction increases the frequency of free-oscillation of

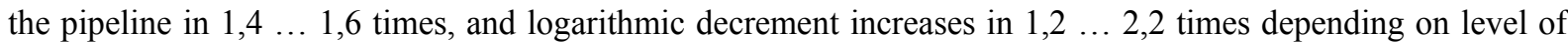
filling, presence or absence of internal superfluous pressure and parameters of prestressed in comparison with pipelines of the traditional constructional decision.

Character of change of frequencies and decrements of oscillation models of prestressed pipeline depending on force tension of the winding thread has shown, that change of force of pretension from $0,25 S_{c r}$ to $0,75 S_{c r}$ leads to increase of frequency of the basic tone of oscillation of models on the average in 1,5 . 1,6 times, and logarithmic decrement of oscillation in 1,2 . 1,25 times that is explained by the level increase of prestressed effect of inclusion of winding threads in teamwork with a wall increases.

\subsection{Results of Experimental Studies at Forced-Oscillation Regime}

In experimental researches of pipeline models at its horizontal forced-oscillation regime the task of the definition of amplitude-frequency characteristics of models taking into account various operating conditions and prestressed parameters, and also an estimation of features of dynamic behavior of prestressed pipelines in comparison with pipelines without winding is assigned.

The analysis of amplitude-frequency dependences has shown essential influence of prestressed to amplitude and frequencies of the forced-horizontal oscillation of the pipeline. So, the increase in values of frequencies of the forced oscillations both on the first and on the second forms of resonant oscillations on average in $1,2 \ldots 1,5$ times at horizontal oscillations and in 1,2 .. 1,6 times at vertical oscillations depending on operational conditions and a step of the cast wire was observed. And the greatest values of increase in frequencies correspond to model of previously strained pipeline with a winding step cast equal d. Frequencies of the forced

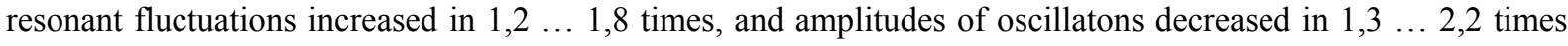
depending on the level of filling, existence or lack of internal excessive pressure and parameters of preliminary tension, in comparison with test data of pipelines without winding.

Amplitude of oscillations of model of previously strained pipeline decreased on average $1,2 \ldots 1,8$ times at horizontal oscillations and in 1,1 . 1,8 times at vertical oscillations, both on the first, and on the second forms of resonant oscillations, depending on filling of the pipeline, internal excessive pressure in the pipeline and a 
winding thread step cast. It is established that nature of oscillations of models of previously strained pipeline corresponds to a form of oscillations of the beam of constant section jammed on the ends. Tests of model of prestressed pipeline under internal pressure has shown, that schedules of amplitude-frequency characteristics of model have smoother character in comparison with a variant of absence of internal pressure.

Tests results of models are shown in drawing 2.

\subsection{Results of Theoretical Studies}

In theoretical part of work tasks about own fluctuations of previously strained pipeline of model of the ring which is pressed out by a winding and on spatial model are solved. The following expressions for determination of own frequencies of oscillations are received:

for the model of the ring that is pressed out by a winding:

$$
\omega=\frac{i\left(i^{2}-1\right)}{r^{2}} \sqrt{\frac{\left(E_{1} \delta_{1}^{3}+E_{2}\left(\delta_{2}+\frac{S_{n \mu}}{a \sigma_{c r}}\right)^{3}\right)}{12 \rho\left(F_{1}+F_{2}\right)\left(i^{2}+1\right)\left(1-\mu^{2}\right)}}
$$

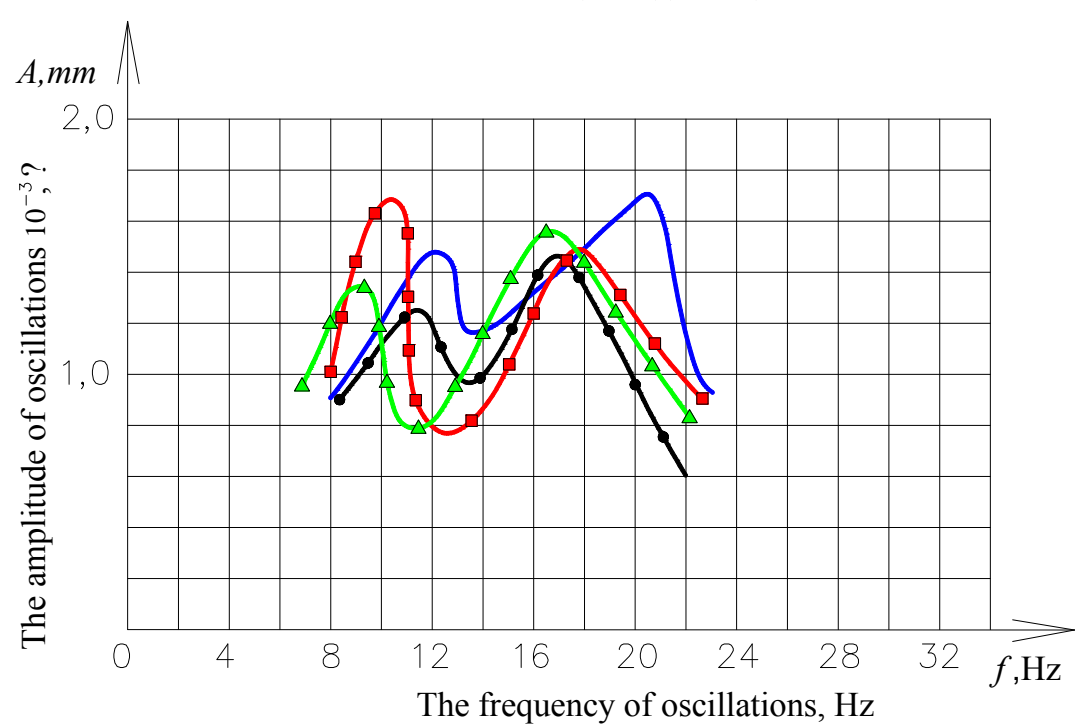

$$
a=0
$$

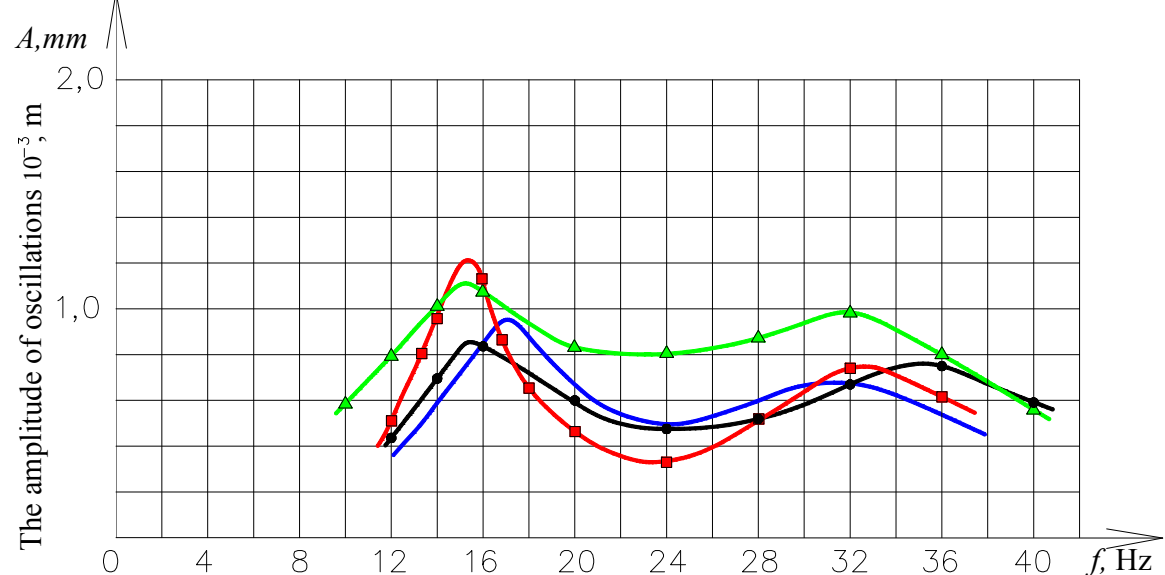

The frequency of oscillations, $\mathrm{Hz}$

$$
a=d
$$




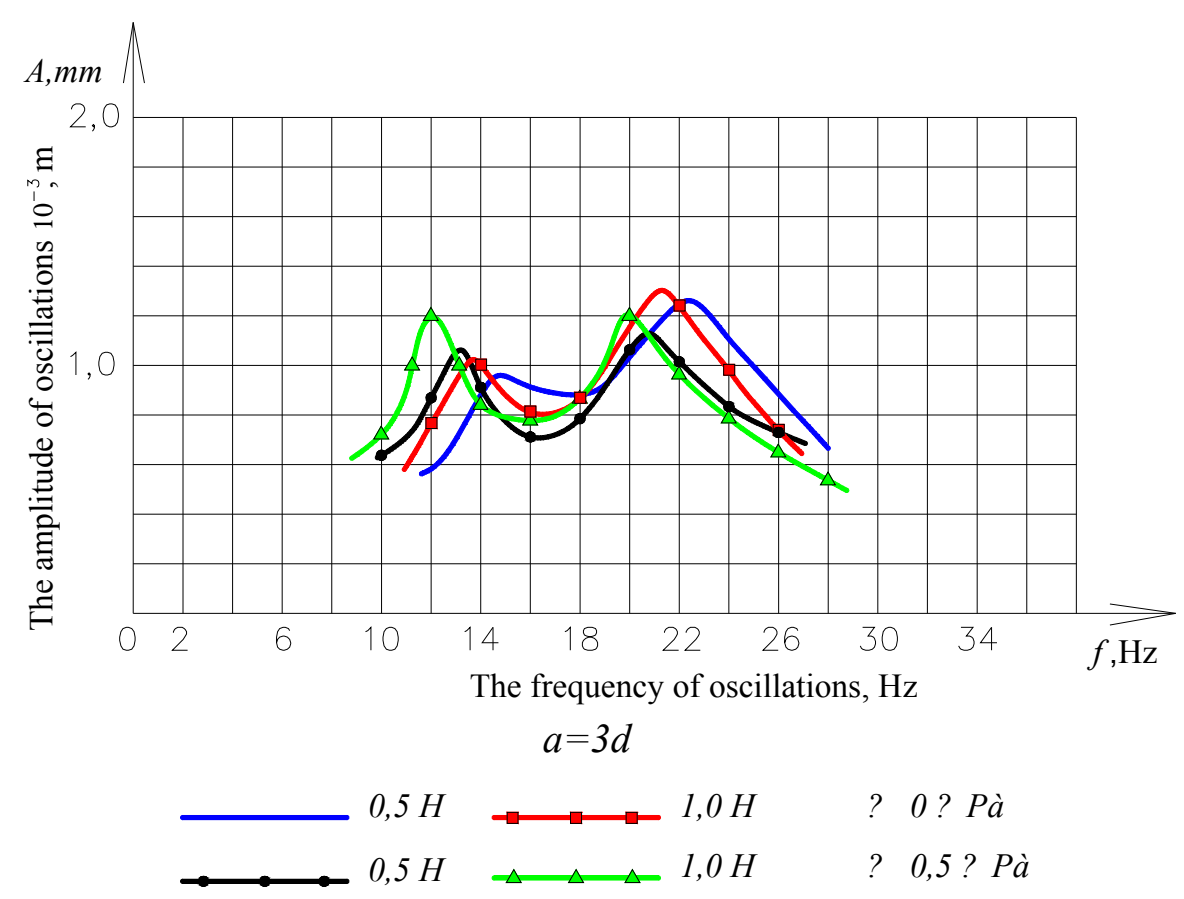

Figure 2. Amplitude-frequency characteristics of model oscillation

for spatial model:

$$
\omega_{n}=\sqrt{\frac{E_{1} \delta_{1}+E_{2}\left(\delta_{2}+\frac{S_{n \mu}}{a \sigma_{c r}}\right)}{2\left(1-\mu^{2}\right) \cdot \rho\left(\delta_{1}+\delta_{2}\right)}\left(\frac{k^{2} r^{2}-1}{r^{2}}+\frac{k}{r} \sqrt{k^{2} r^{2}+\frac{1}{k^{2} r^{2}}+2\left(1+2 \mu^{2}\right)}\right)}
$$

In formulas (1) and (2) $\mathrm{i}$ - number of waves in a circle, $k=\frac{n_{1} \pi}{l}$ - wave number; $E_{1}, E_{2},-$ modules of elasticity of materials of a shell and winding; $F_{1}, F_{2}$ - - the areas of cross section of a ring and a winding; $\delta_{1}$, $\delta_{2}$ - thickness of a wall of a shell and winding; $\mathrm{r}$ - radius of a shell (pipeline); $a$ - winding thread step; $S_{n H}$ effort of a tension of a thread of a winding; $\sigma_{c r}$ - the critical tension of loss of stability; $\rho$ - weight of a shell and winding of single width; $\mu$ - Poisson's coefficient.

Results of calculation on shell model and on model of a ring are given in Table 1.

Table 1. Results of calculation of frequencies of own oscillaions of models of previously strained pipeline

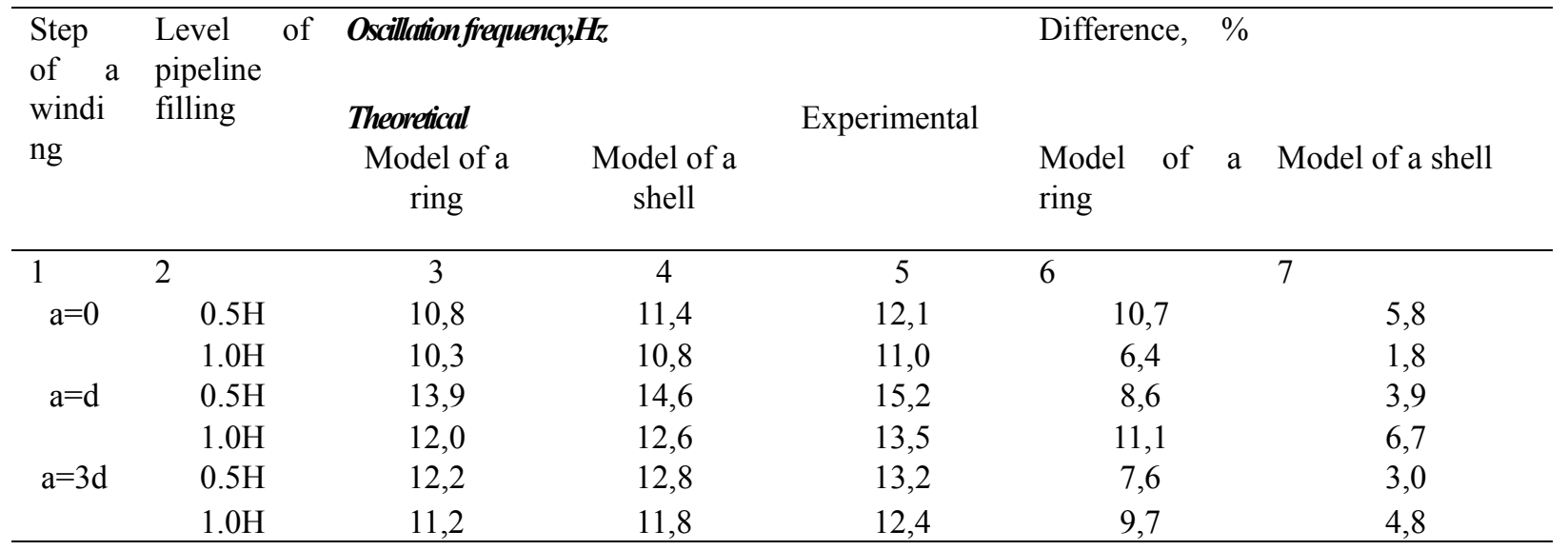


It is revealed that use in calculations of own frequencies of fluctuations of prestressed pipelines of spatial model allows to take into account the influence of shell effects.

It is found that operating conditions of previously strained pipeline are influenced by winding material, its thickness, a preliminary stress and a tilt angle, and parameters of material and thickness of a pipe can accept only the fixed values as they are standardized.

It is shown that due to alignment of size of preliminary stress and an angle of winding it is possible to regulate more flexibly distribution of stress in a wall and a limit condition of the pipeline.

Economic efficiency. Economic rationality of prestressed shell is defined by the relation of total thickness of a shell $\delta_{1}$ and a winding $\delta_{2}$, to thickness of the similar shell executed without preliminary stress $\delta_{0}$ :

$$
\psi=\frac{\delta_{1}+\delta_{2}}{\delta_{0}}=\frac{k_{R}-1}{\left(k_{R}-\alpha_{E}\right)\left(\frac{\sigma_{01}}{R_{1}}+1\right)}+\frac{1-\alpha_{E}}{k_{R}-\alpha_{E}}
$$

On a formula (3) Dependence of impairment of previously strained pipeline at $\alpha_{\mathrm{E}}=1$ from the preliminary stress $\sigma_{01} / \mathrm{R}_{1}$ and parameter $\mathrm{k}_{\mathrm{R}}$ characterizing winding durability is received according to Figure 3 .

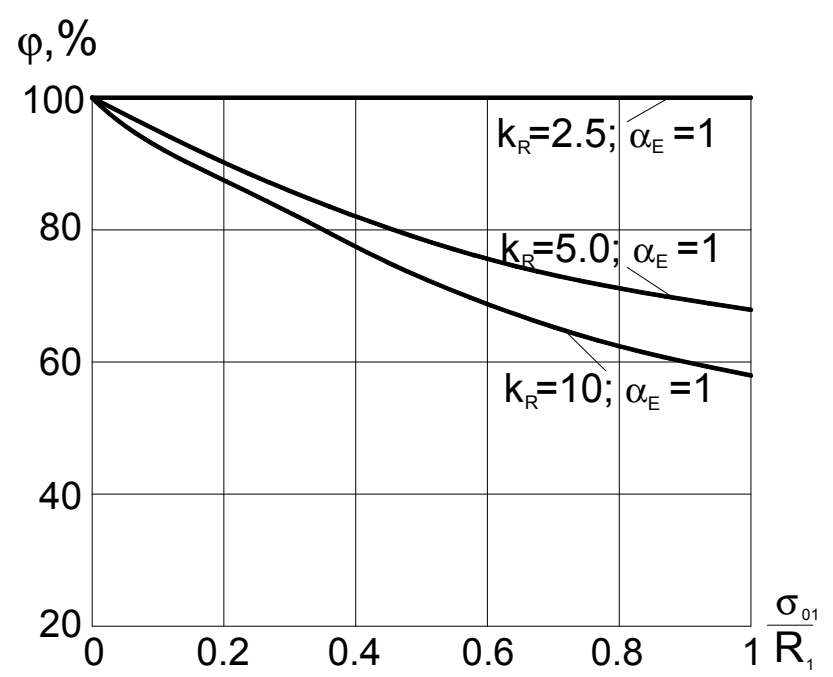

Figure 3. Dependence of cost on relative preliminary stress at various values of durability of a winding

It is visible from the Figure that already at $k_{R}=2.5$ pipeline cost at any value of preliminary stress remains equal to the cost of the pipeline of an usual design without winding. If we take into account that the consumption of metal decreases at increase in the preliminary tension (Figure 3), it is possible to consider effective using as a material winding with parameter $k_{R}=2.5$.

At $k_{R}>2.5$ the cost of the pipeline decreases with increase in preliminary stress, and the decrease is bigger, than $k_{R}$ takes more value.

\section{Discussion of Results}

Comparison of test data for static loading of previously strained pipelines with various step of a winding shows lack of qualitative differences in nature of distribution of deformation of a wall and stress in ring section. However in model of previously strained pipeline with a winding step equal to $3 \mathrm{~d}$ the increase in stress and deformation of a wall of the pipeline in comparison with tests data of the pipeline with a winding step equal to $d$ is observed.

A number of essential advantages of similar designs is established by experimental studies of models of previously strained pipelines on dynamic influences. First, at oscillations of previously strained pipelines the tendency to alignment their movement is observed.

Secondly, improvement of dynamic characteristics of previously strained pipeline in comparison with pipelines of the traditional constructive decision is observed. So, the frequency of the main tone of own oscillations of previously strained pipeline to 1,8 times is higher than the frequency of the main tone of oscillations of the ordinary pipeline, values of logarithmic decrement of oscillations increase on average to 2,2 times, and 
amplitude of oscillations decreases to 2 times.

Thirdly, previously strained pipelines can be effectively used in seismic countries. It is known if the frequency of own oscillations on the values coincides with frequencies of seismic influence, the design gets to a zone of resonant oscillations, long time are there, have big scope that leads to considerable destructions.

Previously strained pipelines build up themselves from resonant frequencies, and bring out of the mode of resonant oscillations. Scope of their fluctuations in $2 \ldots 4$ times is less, and they make only some oscillations as have quickly fading character. So, regulating parameters of preliminary tension it is possible to build up own frequency of the pipeline from dominant frequencies of oscillations of soil. In that case preliminary tension can be considered as active seismic protection of pipelines on the one hand and as a way of regulation by dynamic characteristics with another.

Formulas for determination of frequencies of own oscillations of the pipeline considering parameters of preliminary stress were obtained by the solution of problems of own oscillations of previously strained pipeline on rod model, model of the ring which is pressed out by a winding and model of an elastic thin-walled shell. It is shown that use of shell model more precisely defines value of frequencies in comparison with experimental data. However, it should be noted, for establishment of approximate value also the rod model and model of the ring which is pressed out by a winding are applicable. And applicability of this or that model depends on the pipeline length relation to its diameter.

\section{Conclusion}

Results of experimental studies of models of pipelines on dynamic influences showed that preliminary stress by a high-strength winding possesses the considerable static durability and the improved dynamic characteristics in comparison with pipelines of an usual design.

This technique can be used as a technique of seismic protection of designs. Also this technique allows to regulate dynamic characteristics of a design by selection of parameters of prestressing: step and effort of a tension of a thread.

The formulas of determination of frequencies of free oscillations of the ring which is previously strained by a winding and the extended pipeline with use of the moment less theory of shells considering parameters of preliminary stress and service conditions are offered.

Perspective the directions of researches of previously strained designs can be use of the offered idea as active seismic protection of various sheet designs: tanks, gas-holders, pipelines, silos.

Economic efficiency of use of preliminary tension in pipeline designs is theoretically proved. It is shown that the indicator of decrease in a consumption of material and depreciation generally depends on the level of preliminary stress and indirectly on strength characteristics of materials of a winding and the pipeline.

\section{Acknowledgements}

Work is performed according to the contract for performance of research works within the state order on the "Grant Financing of Scientific Research" program with Committee of Science of the Ministry of Education and Science of the Republic of Kazakhstan.

Authors express gratitude to the staff of laboratory of Mechanical testing South Kazakhstan State University for assistance in carrying out model tests.

\section{References}

Ainabekov, A. I., Arapov, B. R., \& Suleymenov, U. S. (2003). Operation of the elevated prestressed pipelines at operational loadings. Science and education of South Kazakhstan, 35, 12-14.

Andreyev, L.V., Reshetilova, A. P., \& Ruzin V. I. (1986). Influence of prestressing to the size of limit pressure of a cylindrical shell. Problems of durability, 1, 86-91.

Belenya, E. I. (1975). The prestressed bearing metal designs. M., Stroiizdat, y. 416 p.

Belenya, E. I., Astryab, S. M., \& Ramazanov, E. B. (1979). The prestressed metal sheet desogns. M., Stroiizdat, y., $192 \mathrm{p}$.

Building regulations. (2001). 2.04.12.-86. Calculation for durability of steel pipelines. - M.: 12p.

Building regulations. (1985). 2.05.06 - 85* Main pipelines. Design standards. M., 52 p.

Elnashai, A. S., \& Di, Sarno, L. (2008). Fundamentals of Earthquake Engineering. 
Ostsemin, A. A., \& Zavarukhin, V. Yu. (1990). Durability of banded oil pipeline at an inclined winding. Problems of durability, 11, 100-105

Pukhovskiy, A. B. (1984). To a question of increase of seismic stability of metal designs by prestressing. Construction and architecture, 4, 10-13.

Shapovalov, L. A. (1990). Modeling in mechanics of structural elements. M.: 288p.

Smirnov, A. I. (1983). About the effectiveness of banding pipes and cylindrical containers. Problems of durability, 12, 77-79.

Suleymenov U.S., Moldagaliev A.B., Zhanabay N.Zh., Ukibayev M.K. (2005). Prospects of use of prestressing in metal designs of oil-processing industry. Science and education of South Kazakhstan, 3(43), 51-53.

Voevodin, A. A. (1989). Prestressed systems of designs elements. M.: C Stroiizdat,. 298.

Zavarukhin, V. Yu., \& Ostsemin A. A. (1990). To the calculation of the limit state banded pipe. Problems of durability, 1, 76-81.

\section{Copyrights}

Copyright for this article is retained by the author(s), with first publication rights granted to the journal.

This is an open-access article distributed under the terms and conditions of the Creative Commons Attribution license (http://creativecommons.org/licenses/by/3.0/). 